\title{
A pilot study to assess the sensitivity and specificity of an intrasound device in the diagnosis of ankle fractures
}

\author{
Angela Dancocks, Andrew Rouse, John Hiscox
}

\begin{abstract}
Objective-To investigate the use of a simple intrasound device in the detection of malleolar fractures in the accident and emergency (A\&E) department.

Methods-Patients aged 16 to 70 years with ankle injuries presenting within 24 hours were considered for inclusion in the study. Those patients with bony tenderness, inability to weight bear, and swelling were examined with a simple intrasound device before $x$ ray examination. A positive result of discomfort, pain, withdrawal response, or combinations of these was noted.
\end{abstract}

Results-The device had a sensitivity of $85 \%$ and a specificity of $52 \%$ in detecting malleolar fractures.

Conclusions-The use of intrasound in the detection of malleolar fractures cannot be relied upon alone, but it may help to limit the number of $\boldsymbol{x}$ rays taken unnecessarily. A further study is proposed to confirm these findings.

(F Accid Emerg Med 1997;14:230-232)

Keywords: malleolar fractures; intrasound; accident and emergency

Accident and

Emergency

Department,

Northampton General

Hospital,

Northampton

A Dancocks

Somerset Health

Authority, Wellsprings

Road, Taunton,

Somerset

A Rouse

Accident and

Emergency

Department, Aberdeen

Royal Infirmary,

Foresterhill, Aberdeen

$\mathrm{J}$ Hiscox

Correspondence to:

Dr Angela Dancocks,

Accident and Emergency

Department, Northampton

General Hospital NHS

Trust, Cliftonville,

Northampton NN1 5BD.

Accepted for publication 28 February 1997
There is general agreement that doctors in accident and emergency (A\&E) departments request too many $x$ ray examinations of injured ankles to exclude fractures. The publication of evidence based clinical guidelines to limit inappropriate radiographs has had limited success. ${ }^{12}$ Furthermore, even with appropriate use of these guidelines many patients without bony injury will have unnecessary $x$ rays. ${ }^{3}$

A paper published in 1993 in the fournal of Hand Surgery suggests a possible approach to further reducing the use of unnecessary $x$ rays. ${ }^{4}$ Finkenberg et al reported that an inexpensive intrasound device could detect occult fractures of the scaphoid more effectively than $x$ ray.

Intrasound-vibration in the range from 20 $\mathrm{Hz}$ to $20000 \mathrm{~Hz}$ - has been used for many years for the relief of pain associated with soft tissue injuries. Intrasound conducts easily through most tissues and bone, and sound waves resonating around a fracture may cause pain. Finkenberg's paper reported that the use of intrasound resulted in $100 \%$ sensitivity and $95 \%$ specificity in the detection of scaphoid fractures. They concluded that this method was easy to use, reliable, safe, and acceptable to patients. This study aims to assess whether

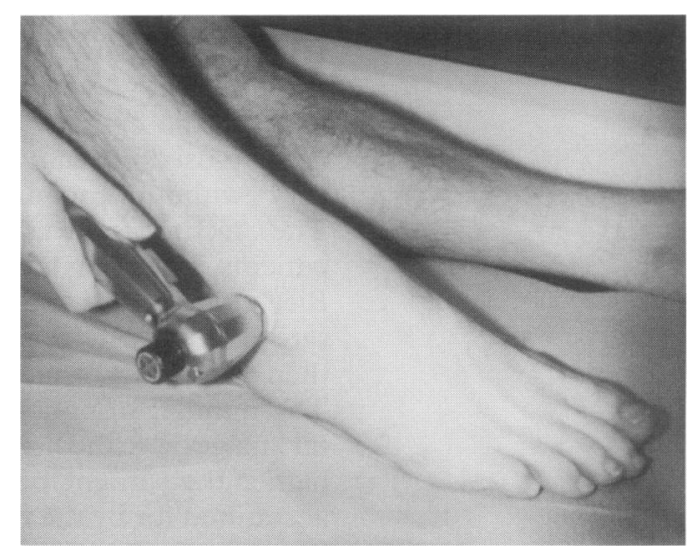

Figure 1 The intrasound device is applied to both malleoli in turn.

there is a role for the intrasound device in the detection of malleolar fractures of the ankle (fig 1).

The objective of this study was to calculate the sensitivity and specificity of an intrasound device in the detection of clinically significant malleolar fractures in patients with ankle injuries presenting to the $\mathrm{A} \& \mathrm{E}$ department.

\section{Methods}

Ethics committee approval was sought before starting the study. A written protocol was agreed by all the authors. All members of participating staff were trained in the use of the intrasound device and assessment of patient response.

It was calculated that an ideal sample size would include 180 subjects.

It was decided that data collection would last 12 months. All patients aged between 16 and 70 years attending the A\&E department at Northampton General Hospital during the study period with a closed injury to their ankle which had occurred within 24 hours were eligible for inclusion in the study. Patients were excluded from the study if they were known to be suffering from diabetes, peripheral vascular disease, neurological disability, or to have had a previous ankle fracture. All patients whom the attending doctor decided required radiographic examination were then entered into the study. The criterion for radiographic examination of the ankle was the presence of bony tenderness of the malleoli, with or without inability to weight bear. Differentiation was not made between tenderness at the tip of the lateral malleolus and at the anterolateral part of the lateral malleolus. Patients gave their consent for inclusion in the study. 
Table 1 Relation between $x$ ray reported fractures and patient response

\begin{tabular}{lcc}
\hline & Fracture & No fracture \\
\hline Irritable, painful withdrawal & 17 & 69 \\
No effect & 3 & 64 \\
Total & 20 & 133
\end{tabular}

Sensitivity $=85 \%$; specificity $=52 \%$.

Both ankles were exposed and examined in turn. The device was run down both the medial and the lateral malleoli as agreed in the protocol. Patients were asked to indicate whether the sensation was uncomfortable, painful, or had no effect. The attending doctor noted any withdrawal response.

All patients then underwent radiographic examination of the injured ankle. Results of the radiographic examination were reported by a consultant radiologist and correlated to the response obtained from the patient.

Patient response was used to calculate sensitivity and specificity.

\section{Results}

Data were collected on 170 subjects. Ages ranged from 16 to 65 years; $52 \%$ were male, $48 \%$ female. Sixteen patients were excluded from the final analysis as the $x$ rays were not available. One patient was excluded as she was given intravenous analgesia before examination.

In 20 patients $(14 \%)$ malleolar fractures were reported on $x$ ray. No patients recorded discomfort on examination of the uninjured ankle. A positive result was accepted as

Inversion/eversion ankle injury in a patient between 16 and 70 without diabetes, peripheral vascular disease or known neurological disorder ?

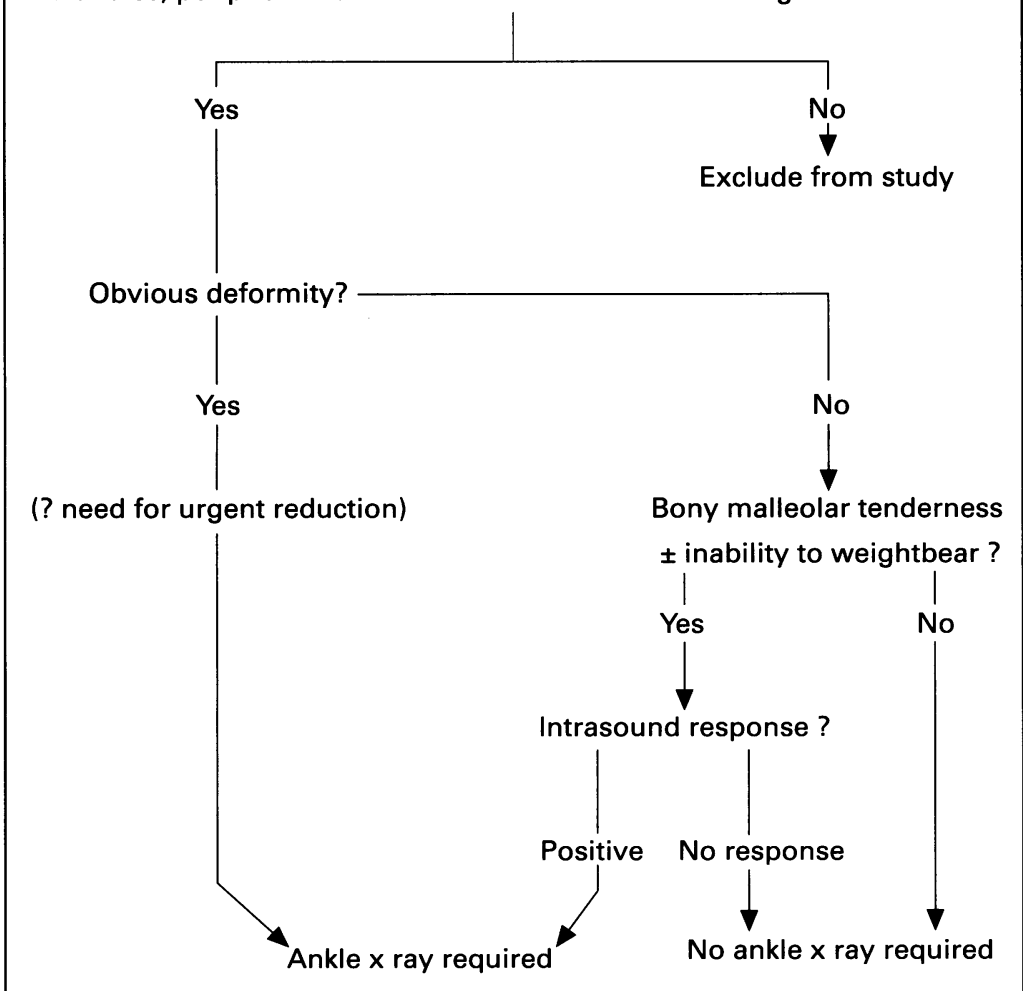

Figure 2 Algorithm determining the use of an intrasound device for ankle injuries seen in the $A \mathcal{E} \cdot E$ department.
Table 2 Relation between $x$ ray reported fractures and withdrawal response

\begin{tabular}{lcc}
\hline & Fracture & No fracture \\
\hline Withdrawal & 3 & 6 \\
Painful irritable, no effect & 17 & 127 \\
Total & 20 & 133 \\
\hline
\end{tabular}

Sensitivity $=15 \%$; specificity $=95 \%$.

discomfort, pain, or a withdrawal response to the application of the intrasound device.

Tables 1 and 2 show the relation between fractures reported on $x$ ray and the patient's response to the intrasound device.

Maximum sensitivity of any test occurs when all abnormal test responses are considered to indicate a true abnormality. The results in table 1 show that the maximum sensitivity of intrasound in diagnosing radiographically demonstrated malleolar fractures in this study was $85 \%$.

Maximum specificity of any test occurs when only the most extreme abnormal test responses are considered indicative of true abnormality. The results reported in table 2 show that the maximum specificity in diagnosing patients without an underlying fracture was $95 \%$.

\section{Discussion}

Ankle injury is one of the most common complaints of patients seen in A\&E departments. The prevalence of radiographic malleolar fractures in this group of patients is relatively low (less than $15 \%) .^{5-7}$ The aim of this study was to determine whether the use of intrasound could be used to decrease the number of $x$ ray requests by identifying those patients without definite malleolar fractures. All patients with bony tenderness in the foot, for example the base of the fifth metatarsal, underwent $x$ ray examination of the foot as well as of the ankle, but the results were not included in this study.

Twenty patients (14\%) with radiographically diagnosed malleolar fractures were identified. Seventeen of these $(85 \%)$ reported discomfort or pain at the fracture site during application of the intrasound device. Sixty four patients who did not have radiographic evidence of a malleolar fracture reported discomfort or pain. Three patients were reported as having fractures who had a "no effect" response. One of these patients had a hairline fracture of the lateral malleolus which was missed by the attending doctor and was recalled after the $x$ rays were reported; the other two patients had avulsion fractures of the tip of the lateral malleolus. The detection of the avulsion fractures did not change the management of these patients. The patients were not able to localise the exact origin of the pain or discomfort produced and therefore it was not possible to differentiate an avulsion fracture from a more proximal fracture.

This study shows that the sensitivity and specificity of the intrasound device in the detection of radiographic fractures are too low for the device to be used alone in clinical practice to exclude or diagnose a malleolar fracture. However, if only patients with a positive 
response - that is, withdrawal, pain, or discomfort-had been $x$ rayed the total number of $x$ rays would have been reduced by 67 , a reduction of $44 \%$ of the total number of $x$ rays undertaken. Only one clinically significant fracture was missed and this was not detected by the attending doctor at the first visit and did not alter the initial management of the patient.

These results compare favourably with the study by Stiell et al, who noted a $28 \%$ reduction in the number of $x$ rays taken after the introduction of the Ottawa ankle rules for identifying patients for $x$ ray examination. ${ }^{8}$ The Ottawa rules identify patients with specific malleolar tenderness or inability to bear weight, and studies have shown that the use of these rules does not result in any clinically significant fractures being missed. Our study included all patients with any malleolar tenderness and did not differentiate between tenderness at the tip of the lateral malleolus, the anterolateral malleolus, or the posterior malleolus, as did the Ottawa study.

CONCLUSION

The sensitivity and specificity of the intrasound device are too low for the detection of clinically significant malleolar fractures alone. However, the use of an inexpensive intrasound device may be of value in decreasing the overall number of $x$ rays taken by staff in $A \& E$ to exclude ankle fractures, by identifying those patients with a negative response. Further larger studies are required to confirm these findings.

We propose that use of the intrasound device with the introduction of the algorithm shown in fig 2 would significantly reduce the total number of $x$ rays taken of ankle injuries in A\&E.

1 Brooks SC, Potter BT, Rainey JB. Inversion injuries of the ankle; clinical assessment and radiographic review. BMJ $1981 ; 282: 607-8$.

2 Dunlop MG, Beattie TF, White GK, Raab GM, Doull RI. Guidelines for selective radiological assessment of inversion ankle injuries. BMJ 1986;293:603-5.

3 Anis AH. Cost-effectiveness analysis of the Ottawa ankle rules. Ann Emerg Med 1995;26:4.

4 Finkenberg JG, Heffer E, Kelly C, Zinar DM. Diagnosis of occult scaphoid fractures by intrasound vibration. J Hand Surg [Am] 1993;18A:4-7.

5 Vargish $T$, Clarke WR, Young RA, Jensen A. The ankle injury-indications for the selective use of $x$-rays. Injury 1983;6:507-12.

6 Royal College of Radiologists Working Party. Radiography of injured arms and legs in eight $A \& E$ units in England and Wales. BMJ 1985;291:1325-8.

7 Montague AD, McQuillan RF. Clinical Assessment of apparently sprained ankle and detection of fracture. Injury 1985:16.545-6.

8 Stiell IG, McKnight RD, Greenburg GH, McDowell I, Nair RC, Wells GA, et al. Implementation of the Ottawa ankle rules. JAMA 1994;271:827-32.

\section{THE FACULTY OF ACCIDENT AND EMERGENCY MEDICINE}

Applications are invited from Consultants in Accident and Emergency Medicine who have been in post for at least five years and who wish to be appointed to the Panel of Examiners. The examination for the Exit Diploma of FFAEM is currently held twice a year at various venues in the United Kingdom.

\section{The closing date for receipt of applications is 1st September 1997}

Further details and an application form are available from:

The Faculty of Accident and Emergency Medicine

$$
\text { 35-43 Lincoln's Inn Fields }
$$

London WC2A 3PN

Tel: 01714057071 